\title{
Testing collinear factorization in a spectator model with mass corrections
}

\author{
Juan V. Guerrero* and Alberto Accardi \\ Hampton U. and Jefferson Lab, USA \\ E-mail: juanvg@jlab.org
}

\begin{abstract}
In perturbative $\mathrm{QCD}$, the masses of the hadrons involved in high energy reactions can usually be neglected. However, in the case of Kaon production in electron-proton collisions at low (and not so low) beam energies this may not be a good approximation. In particular, a recent proposal to include hadron masses in theoretical calculations shows how these Hadron Mass Corrections (HMCs) can explain a large discrepancy observed in measurements performed at the HERMES and COMPASS experiments. In this talk, we present preliminary results of a spectator model calculation designed to test the range of validity of the approximations needed in the proposed factorization scheme. We focus on inclusive DIS scattering as a first step towards the analysis of HMCs in semi-inclusive processes.
\end{abstract}

XXVII International Workshop on Deep-Inelastic Scattering and Related Subjects - DIS2019

8-12 April, 2019

Torino, Italy

${ }^{*}$ Speaker. 


\section{Introduction}

Collinear Parton Distribution Functions (PDFs) in QCD encode information regarding the longitudinal quark and gluon structure of hadrons and can be accessed through hard scattering reactions. This takes advantage of QCD factorization theorems, such as Collinear Factorization $(\mathrm{CF})$, which are usually formulated in the asymptotically large limit of some physical scale, e.g. the photon virtuality $Q^{2}$ in the case of Deep Inelastic Scattering (DIS). However, experiments at low beam energy, such as at Jefferson Lab, involve low photon virtualities, and require theoretical control of $\mu^{2} / Q^{2}$ kinematical power corrections in addition to dynamical higher twist effects. For example, in inclusive DIS $\mu=M$ is the target mass, and Semi Inclusive DIS receives corrections also from the mass of the observed hadron.

These "Hadron Mass Corrections" (HMCs) have been recently explored in Ref. [1, 2], and can even affect relatively high-energy experiments such as HERMES and COMPASS, possibly explaining the apparent large discrepancy between their measurements of integrated kaon multiplicities [2, 3]. At Jefferson Lab, mass effects become large also for pion production. In this talk we present a model calculations designed to test the validity of the sub-asymptotic kinematic approximations needed in the Hadron Mass Correction Scheme [3]. We initially focus on inclusive DIS in order to avoid complications due to the non trivial interplay of initial and final state kinematics in semi-inclusive processes discussed in Ref. [2].

\section{DIS in a spectator model}

The DIS kinematics is defined in Fig. 1 left. In the final state, the remnant $X$ and the recoil quark momentum $k^{\prime}$ are not measured. However, we assume the identity of the latter can be experimentally determined, in analogy with measurement of the charm-tagged $F_{2}^{c}$ structure function, and we assume the quark mass $m_{q}$ to be known.

The four-momenta of the external particles and target quark can be parametrized in terms of light cone unit vectors $n$ and $\bar{n}$, with $n^{2}=\bar{n}^{2}=0$ and $n \cdot \bar{n}=1$. In the so called " $(p, q)$ frame" [1], in which the target and virtual photon are coplanar, with zero tranverse momentum $\left(\boldsymbol{p}_{\boldsymbol{T}}=\boldsymbol{q}_{\boldsymbol{T}}=\mathbf{0}\right)$

$$
p^{\mu}=p^{+} \bar{n}^{\mu}+\frac{M^{2}}{2 p^{+}} n^{\mu}, \quad q^{\mu}=-\xi p^{+} \bar{n}^{\mu}+\frac{Q^{2}}{2 \xi p^{+}} n^{\mu}, \quad k^{\mu}=x p^{+} \bar{n}^{\mu}+\frac{k^{2}+\boldsymbol{k}_{\perp}^{2}}{2 x p^{+}} n^{\mu}+k_{\perp}^{\mu},
$$

where $\xi \equiv-\frac{q^{+}}{p^{+}}=\frac{2 x_{B}}{1+\sqrt{1+4 x_{B}^{2} M^{2} / Q^{2}}}$ is the so-called Nachtmann scaling variable, $x_{B}=\frac{Q^{2}}{2 p \cdot q}$ is the Bjorken scaling variable, and $x=\frac{k^{+}}{p^{+}}$is the light-cone momentum fraction carried by the parton ${ }^{1}$.

Our goal is to mimic and simulate the real electron-proton DIS ocurring in nature with a simpler, calculable toy model. For this, we use an idealized field-theory $[4,5]$ with a spin $1 / 2$ particle representing a nucleon of mass $M$, an active quark of mass $m_{q}$, and a scalar diquark "spectator". At LO, the latter describes target fragmentation, which is a complex process in QCD, with a single particle of mass $m_{\phi} \sim\left\langle m_{X}\right\rangle$. In this "spectator model", the nucleon, quark and the spectator interact through a vertex $\mathscr{Y}=i g\left(k^{2}\right) \mathbb{I}$, with $g\left(k^{2}\right)$ a dipolar form factor:

$$
g\left(k^{2}\right)=g \frac{k^{2}-m_{q}^{2}}{\left|k^{2}-\Lambda^{2}\right|^{2}}
$$

\footnotetext{
${ }^{1}$ The "plus" and "minus" components $a^{\mu}$ are defined by $a^{+}=a \cdot n=\left(a^{0}+a^{3}\right) / \sqrt{2}$ and $a^{-}=a \cdot \bar{n}=\left(a^{0}-a^{3}\right) / \sqrt{2}$.
} 

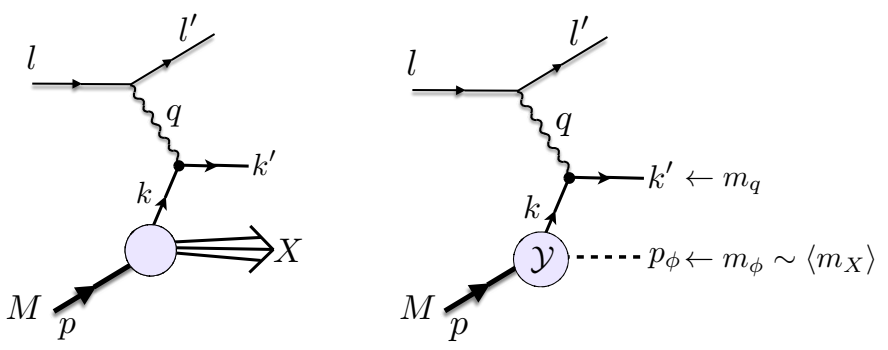

Figure 1: Electron-proton DIS kinematics Left diagram: real one, where $X$ is remnant of the target fragmentation. Right diagram: spectator model, with remnant replaced by a scalar diquark.

where $g$ is an appropriate coupling constant (playing no role in the present discussion) and the parameter $\Lambda$ cuts off ultraviolet divergences in $k^{2} \gg \Lambda^{2}$ [4]. This cutoff also imposes a minimal length scale of $\mathscr{O}(1 / \Lambda)$, and effectively simulates confinement in the nucleon target.

In contrast to the real electron-proton scattering, in the spectator model it is possible to calculate the hadronic tensor in an exact way. At the lowest order in $g$, this receives contributions from the 3 processes depicted in Fig. 2: photon-quark scattering, which mimics DIS; photo-excitation of the proton with subsequent decay into a quark and a spectator; and interference between these two.

The contribution of each diagram in Fig. 2 is not gauge invariant by itself, but the sum is. Since each diagram contains different physics, we can use parity invariant projectors $[6,7,8]$ to extract their individual gauge invariant structure functions; for example,

$$
F_{1, \text { g.i. }}^{\text {DIS }}=\frac{1}{2}\left(-\hat{g}^{\mu v}+\frac{\hat{p}^{\mu} \hat{p}^{v}}{\hat{p}^{2}}\right) W_{\mu v}^{\mathrm{DIS}}
$$

where $\hat{p}^{\mu}=p^{\mu}-\frac{p \cdot q}{q^{2}} q^{\mu}, \hat{g}^{\mu v}=g^{\mu v}-\frac{q^{\mu} q^{v}}{q^{2}}$, and $W_{\mu \nu}^{\mathrm{DIS}}$ is calculated from the diagram in Fig. 2a. Here we focus on the DIS piece because collinear factorization is intended to provide a controlled approximation only to this contribution. The role of the other two diagrams is discussed in the talk's slides and in Ref. [8].

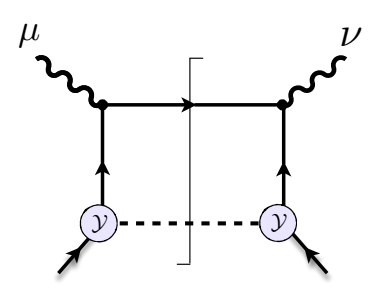

(a) DIS

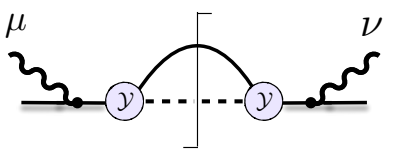

(b) "Resonance"

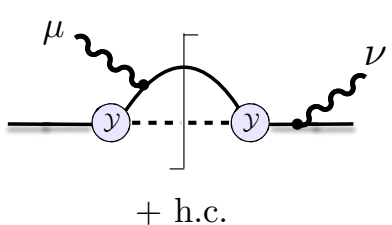

(c) Interference

Figure 2: Diagrams contributing to $e p$-scattering up to order $g^{2}$.

\section{Collinear factorization for DIS in the spectator model}

In the model, it is also possible to calculate the structure function with the collinear approximation used to analyze real life DIS: $F_{1} \approx F_{1}^{\mathrm{CF}}=\int \frac{d x}{x} \mathscr{H}_{1}(x) q(x)$, where $\mathscr{H}_{1}=2 \pi e_{q}^{2} x \delta(x-\bar{x})$ 
is the tree level hard scattering coefficient, and $q(x)=\int d^{2} \boldsymbol{k}_{\boldsymbol{T}} d k^{-} \operatorname{Tr}\left[\beta^{2}\right]$ is the PDF, also calculable in the model! The delta function is a consequence of four-momentum conservation in the hard scattering vertex and the top cut in Fig. 2(a). Finally,

$$
F_{1}^{\mathrm{DIS}}\left(x_{B}, Q^{2}, \mu\right) \approx F_{1}^{\mathrm{CF}}\left(x_{B}, Q^{2}, \mu\right)=2 \pi e_{q}^{2} q\left(\bar{x}, Q^{2}, \mu\right),
$$

where $\bar{x}$ approximates the light cone fraction for the incoming quark determined by the hard scattering kinematics in the full diagram. This reads

$$
x=\xi\left(1+\frac{m_{q}^{2}+k_{T}^{2}}{Q^{2}}-\frac{\left(m_{q}^{2}+k_{T}^{2}\right)\left(k^{2}+k_{T}^{2}\right)}{Q^{4}}+\mathscr{O}\left(\frac{\mu^{6}}{Q^{6}}\right)\right),
$$

and depends only on two mass scales, namely, the scattered transverse quark mass $m_{q T}^{2}=m_{q}^{2}+k_{T}^{2}$ and the "light cone virtuality" of the incoming parton, $v^{2}=k^{2}+k_{T}^{2}$, that measures how different from zero $k^{-}=v^{2} /\left(2 k^{+}\right)$is.

In the full diagram the unobserved $k^{2}$ would be fixed by the bottom cut in Fig. 2(a), but neither this nor the equally unobserved $k_{T}^{2}$ can be controlled in an inclusive process and we have to resort to a kinematic approximation to determine $x$. Nonetheless, we need only to worry about the latter, or rather only about $m_{q T}$, since $v^{2}$ (and therefore $k^{2}$ ) only contributes at $\mathscr{O}\left(1 / Q^{4}\right)$ and is

\begin{tabular}{c|cc|c}
$\bar{x}$ & $M$ & $m_{q}$ & $k_{T}^{2}$ \\
\hline$x_{B}$ & 0 & 0 & 0 \\
$\xi$ & $\checkmark$ & 0 & 0 \\
$\xi\left(1+\frac{m_{q}^{2}}{Q^{2}}\right) \equiv \xi_{q}$ & $\checkmark$ & $\checkmark$ & 0 \\
$-\bar{\xi}\left(1+\frac{m_{q}^{2}+\left\langle k_{T}^{2} \bar{T}\right.}{Q^{2}}\right) \equiv \overline{\xi_{q}^{(T)}}$ & $\checkmark$ & $\checkmark$ & $\checkmark\left\langle k_{T}^{2}\right\rangle$
\end{tabular}

Figure 3: Kinematical approximations for $x$. negligible in DIS kinematics. We can identify three (plus one) $x \approx \bar{x}$ approximations depending on the kinematic variables we decide to neglect: see the table in Fig. (3), where the approximation above the dashed line include only external, experimentally observable quantities, and below the line we considered the most relevant internal variable, namely, $k_{T}^{2}$, albeit only on average.

Despite the fact that $v^{2}=k^{2}+k_{T}^{2}$ and $k_{T}^{2}$ are internal variables and cannot be directly measured in inclusive processes, we can compute their average values in the model: $\langle\mathscr{O}\rangle\left(x_{B}, Q^{2}\right)=$ $\frac{\int_{0}^{k_{T}^{2}, \max } d k_{T}^{2} d k^{2} d x \mathscr{O}\left(x, k_{T}^{2}, k^{2}\right) \mathscr{F}_{1}^{\mathrm{DIS}}\left(x, k_{T}^{2}, k^{2}\right)}{\int_{0}^{k_{T, m a x}} d k_{T}^{2} d k^{2} d x \mathscr{F}_{1}^{\mathrm{DIS}}\left(x, k_{T}^{2}, k^{2}\right)}$, where $k_{T, \text { max }}^{2}$ is determined by the available invariant mass and the final state particle masses (the virtuality $k^{2}$ is determined at LO by the lower cut in Fig. 1a), $\mathscr{O}\left(x, k_{T}^{2}, k^{2}\right)$ is a generic observable, and $\mathscr{F}_{1}^{\text {DIS }}$ is the unintegrated structure function. The dependence on $x_{B}$ and $Q^{2}$ are due to the external kinematics, which is left understood on the right hand side. In collinear factorization, momentum conservation in the transverse (and minus) directions is neglected in order to reduce the loop integration only to the plus light cone direction, and the averages read $\langle\mathscr{O}\rangle_{\mathrm{CF}}\left(x_{B}, Q^{2}\right)=\frac{\int_{0}^{\infty} d k_{T}^{2} d k^{2} d x \mathscr{O}\left(\bar{x}, k_{T}^{2}, k^{2}\right) \mathscr{F}_{1}^{\mathrm{CF}}\left(x, k_{T}^{2}, k^{2}\right)}{\int_{0}^{\infty} d k_{T}^{2} d k^{2} d x \mathscr{F}_{1}^{\mathrm{CF}}\left(x, k_{T}^{2}, k^{2}\right)}$ with no limit on $k_{T}^{2}$.

In the left panel of Fig. 4, we show $\left\langle k_{T}^{2}\right\rangle$ as a function of $x_{B}$ for several values of $Q^{2}$, and notice that $\left\langle k_{T}^{2}\right\rangle \sim \mathscr{O}\left(m_{q}^{2}\right)$ is not a priori negligible in Eq. (3.2). In the right panel, we show the average light-cone virtuality $\left\langle k^{2}+k_{T}^{2}\right\rangle$, finding that at small values of $x_{B}$ the parton behaves like a collinear massless parton. It is also clear that the incoming quark virtuality is negative, $\left\langle k^{2}\right\rangle<-\left\langle k_{T}^{2}\right\rangle$, as it should for a bound quark. Thus, the typical parton model approximation $k^{2} \sim 0$ can be quite inaccurate, and should rather be substituted with $k^{-} \sim 0$. 

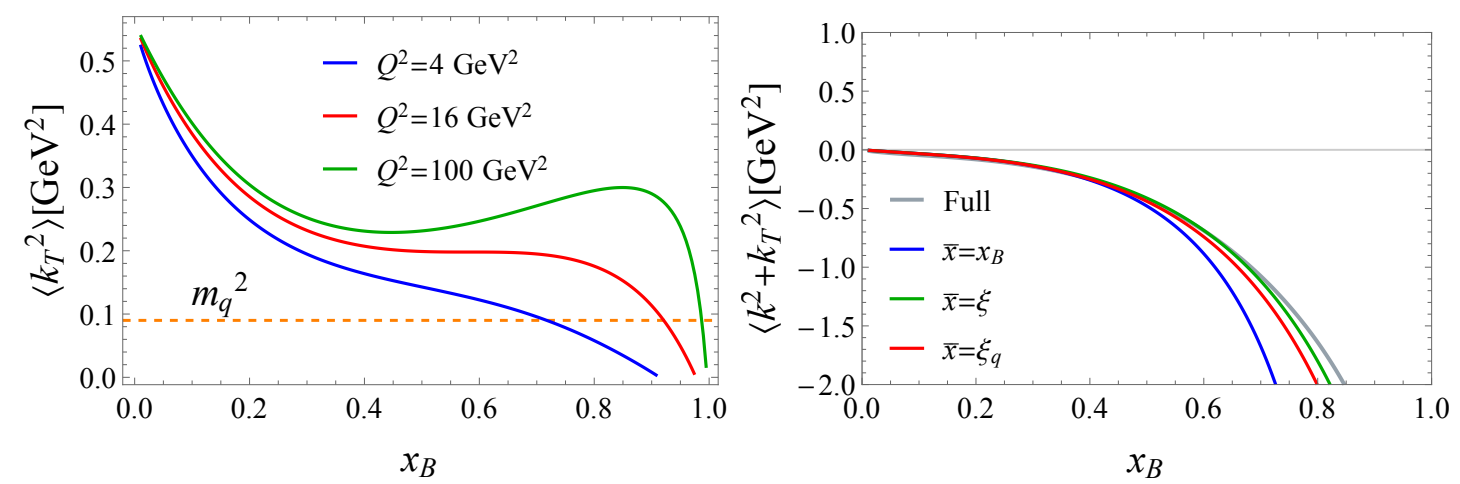

Figure 4: Average "unobserved" kinematics of the incoming quark as a function of $x_{B}$. Left: average $k_{T}^{2}$ in the full model (the orange dashed line indicates the value of $m_{q}^{2}$ for reference). Right: average light cone virtuality $v^{2}=k^{2}+k_{T}^{2}$ in the full model, compared to various collinear kinematic approximations.

\section{Testing factorization}

We can now test the validity of the generalized collinear approximation (3.1) by comparing the exact calculation with the factorized $F_{1}{ }^{\mathrm{CF}}$ for each kinematic approximation listed in Fig. 3 (we use $m_{q}=0.3 \mathrm{GeV}, \Lambda=0.609 \mathrm{GeV}$ and $m_{\phi}=0.822 \mathrm{GeV}$, fitted to known PDFs in Ref. [4]).

In Fig. 5 , we show the ratio of collinear to full $F_{1}$ structure functions for each proposed approximation in Table (3.1), and two different target mass values. The usual, asymptotic choice $x=x_{B}$ in general is not a good approximation, since it depends strongly on the target mass (something already observed in Ref. [5] for $k_{T}$-dependent structure functions). On the other hand, the choice $x=\xi_{q}$ provides the closest approximation to the full $F_{1}$ using only external variables. The effect of the "missing" $k_{T}$ is shown by the dashed-orange line in Fig. 5: the additional $\left\langle k_{T}^{2}\right\rangle / Q^{2}$ correction largely recovers the exact calculation. These transverse-motion-induced power corrections are however outside of the reach of our leading twist calculation, but can likely be handled extending this to higher-twist [9]. Even so, we notice that factorization would break for $x_{B} \gtrsim 0.6$ because it does not respect momentum conservation in the transverse direction.

Finally, in Fig. 6, we compare the collinear $x=\bar{x}$ to the full $\langle x\rangle$. This reinforces the conclusion that $x=\xi_{q}$ is an adequate representation of the parton's longitudinal kinematics, but $x=x_{B}$ is not.
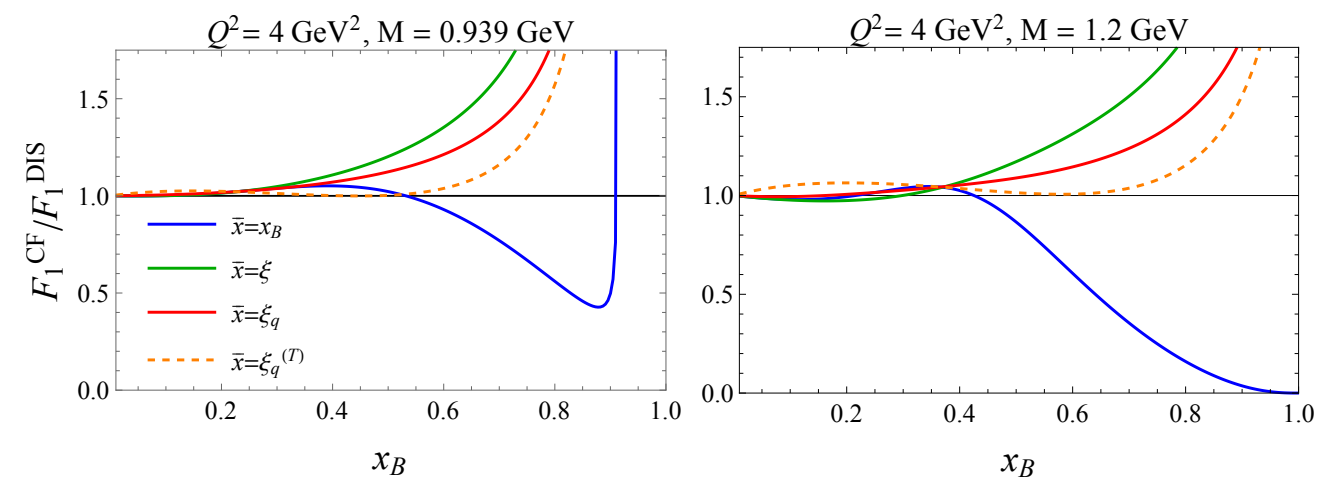

Figure 5: Ratio of collinear to full DIS structure functions. 

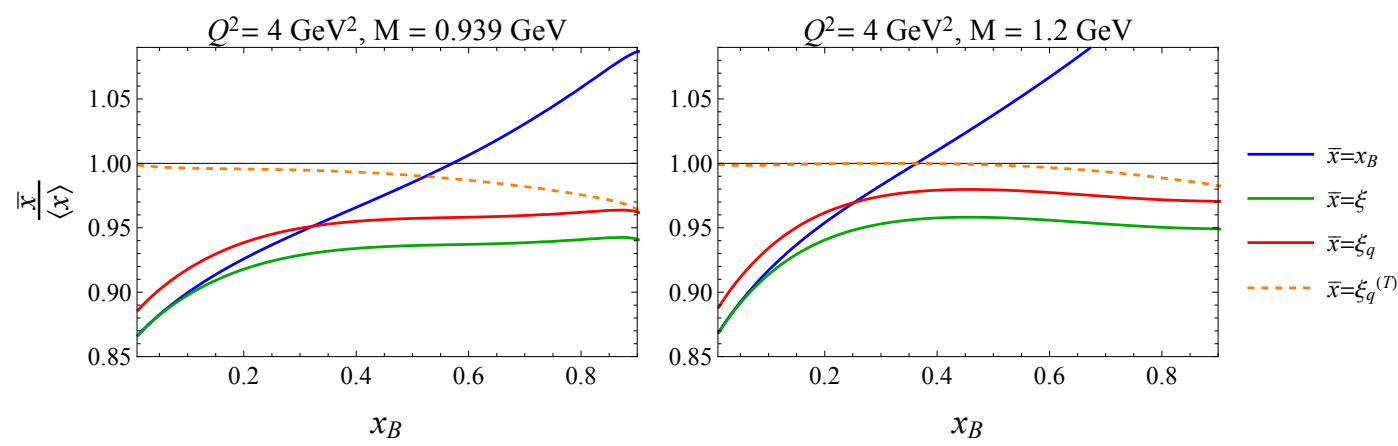

Figure 6: Ratio of approximated $\bar{x}$ to the full $\langle x\rangle$ for two choices of target mass.

In conclusion, in the context of a spectator DIS model, we have verified that the range of validity of collinear factorization can be extended to subasymptotic values of $Q^{2}$ largely by using the mass-corrected scaling variable $\xi_{q}$ instead of $x_{B}$. We have also explicitly illustrated an inherent limitation of collinear factorization, that breaks down at large $x_{B}$ due to the neglect of momentum conservation in the transverse (and light-cone minus) direction.

\section{Acknowledgments}

This work was supported by the DOE contract No. DE-AC05-06OR23177, under which Jefferson Science Associates, LLC operates Jefferson Lab, and No. DE-SC0008791. J.V.G. was also partially supported by the Jefferson Science Associates 2018-2019 Graduate Fellowship Program.

\section{References}

[1] A. Accardi, T. Hobbs and W. Melnitchouk, Hadron mass corrections in semi-inclusive deep inelastic scattering, JHEP 11 (2009) 084 [0907.2395].

[2] J. V. Guerrero, J. J. Ethier, A. Accardi, S. W. Casper and W. Melnitchouk, Hadron mass corrections in semi-inclusive deep-inelastic scattering, JHEP 09 (2015) 169 [150 5 . 02739].

[3] J. V. Guerrero and A. Accardi, Gauge invariance and kaon production in deep inelastic scattering at low scales, Phys. Rev. D97 (2018) 114012 [1711.04346].

[4] A. Bacchetta, F. Conti and M. Radici, Transverse-momentum distributions in a diquark spectator model, Phys. Rev. D78 (2008) 074010 [0807.0323].

[5] E. Moffat, W. Melnitchouk, T. C. Rogers and N. Sato, What are the low- $Q$ and large-x boundaries of collinear QCD factorization theorems?, Phys. Rev. D95 (2017) 096008 [1 702 . 03955 ].

[6] M. A. G. Aivazis, F. I. Olness and W.-K. Tung, Leptoproduction of heavy quarks. 1. General formalism and kinematics of charged current and neutral current production processes, Phys. Rev. D50 (1994) 3085 [hep-ph/9312318].

[7] A. Accardi and J.-W. Qiu, Collinear factorization for deep inelastic scattering structure functions at large Bjorken x(B), JHEP 07 (2008) 090 [0 805 . 1496].

[8] J. V. Guerrero and A. Accardi, Collinear Factorization with hadron masses in a spectator model, in preparation .

[9] J.-W. Qiu, Twist Four Contributions to the Parton Structure Functions, Phys. Rev. D42 (1990) 30. 